\title{
BMJ Open Study protocol for a randomised- controlled study on emotion regulation training for adolescents with major depression: the KONNI study
}

\author{
Ellen Greimel (D , ${ }^{1}$ Lisa Feldmann, ${ }^{1}$ Charlotte Piechaczek, ${ }^{1}$ Frans Oort, ${ }^{2}$ \\ Jürgen Bartling, ${ }^{1}$ Martin Schulte-Rüther (D) , ${ }^{3,4,5}$ Gerd Schulte-Körne ${ }^{1}$
}

To cite: Greimel E,

Feldmann L, Piechaczek C, et al. Study protocol for a randomised-controlled study on emotion regulation training for adolescents with major depression: the KONNI study. BMJ Open 2020;10:e036093. doi:10.1136/ bmjopen-2019-036093

- Prepublication history for this paper is available online. To view these files, please visit the journal online (http://dx.doi org/10.1136/bmjopen-2019036093).

$E G$ and $L F$ are joint first authors.

Received 29 November 2019

Revised 17 June 2020

Accepted 10 July 2020
D) Check for updates

(c) Author(s) (or their employer(s)) 2020. Re-use permitted under CC BY-NC. No commercial re-use. See rights and permissions. Published by BMJ.

For numbered affiliations see end of article.

Correspondence to Dr Ellen Greimel;

Ellen.Greimel@med.unimuenchen.de

\section{ABSTRACT}

Introduction Major depression (MD) often has its onset during adolescence and is associated with significant morbidity and mortality. One important factor for the development and maintenance of adolescent $\mathrm{MD}$ are disturbances in emotion regulation and the underlying neural processes. Cognitive reappraisal (CR) is a particular adaptive emotion regulation strategy. Previously, it has been shown in healthy adults that a task-based training in CR is efficient to reduce negative affect, and that these effects translate into everyday life.

This randomised controlled trial examines for the first time whether a task-based training in CR proves effective in MD adolescents. Specifically, we will investigate whether the CR training improves the ability to downregulate negative affect in MD individuals as assessed by behavioural and neurobiological indices, and whether training effects generalise outside the laboratory.

Methods and analysis Adolescents with MD will be randomly allocated to a group that either receives a task-based training in CR or a control training. Both involve four training sessions over a time period of 2 weeks. In the CR training, participants will be instructed to downregulate negative affective responses to negative pictures via $\mathrm{CR}$, while the control training involves picture viewing. During the training sessions, the Late Positive Potential, gaze fixations on negative picture aspects and affective responses to pictures will be collected. Before and after the training programmes, and at a 2-week follow-up, overall negative and positive affect, rumination and perceived stress will be assessed as primary outcomes. Analyses of variance will be conducted to test the effectiveness of the CR training with regard to both primary outcomes and task-based behavioural and neurobiological parameters.

Ethics and dissemination The study was approved by the Ethics Committee of the Medical Faculty of the LMU Munich, Germany. The results will be published in peer-reviewed journals and disseminated through conferences, social media and public events.

Trial registration details ClinicalTrials.gov NCT03957850, registered 21 $1^{\text {st }}$ May 2019; URL: https:// clinicaltrials.gov/ct2/show/NCT03957850.
Strengths and limitations of this study

- This study is the first to examine the effectiveness of a targeted cognitive reappraisal (CR) training in youths with major depression.

- A randomised controlled design is employed including an active control group.

- In addition to behavioural and questionnaire data, objective neurobiological indices will be collected to assess the effectiveness of the CR training.

- If proven effective, the CR training can be a promising and cost-effective approach to complement and enhance the efficacy of established treatments for adolescent major depression.

- As the follow-up is limited to 2 weeks, the long-term effectiveness needs to be explored in future studies.

\section{INTRODUCTION}

Major depression (MD) is among the most debilitating, costly and common psychiatric disorders worldwide. ${ }^{1}$ The risk of suffering from a depressive episode sharply rises during adolescence, with point prevalence rates of up to $7 \% .^{2}$ Adolescents with MD show deficient emotion regulation (ER) ${ }^{3}$ that is, they have difficulties to modulate emotional responses by initiating appropriate regulatory processes. ${ }^{4}$ Disturbances in ER and the underlying neural processes have been suggested to be an important risk factor for the development and maintenance of MD. ${ }^{35}$ Thus, the development of training regimens that target deficient ER in MD is an important research avenue.

Cognitive behavioural therapy (CBT) belongs to the gold standard treatments in juvenile MD. However, less than $50 \%$ of MD adolescents respond to CBT and only about one-third enter remission after treatment. ${ }^{67}$ Given the debilitating consequences of adolescent $\mathrm{MD},{ }^{8}$ there is an urgent need to enhance the efficacy of established interventions. 
Recent findings in adults with MD suggest that an additional training of ER might be a promising approach to improve treatment effects. ${ }^{910}$ To date, it remains unclear whether these findings can be transferred to juvenile MD. In this regard, the present study takes a first step and systematically investigates the effects of a focused ER training in adolescent MD.

\section{Cognitive reappraisal in depressed individuals}

Cognitive reappraisal (CR) is a frequently studied ER strategy and involves the reinterpretation of a situation such that the emotional response is changed. The habitual use of CR has been shown to relate to good interpersonal functioning and well-being. ${ }^{11}$ Questionnaire studies have found that depressive symptoms are associated with less habitual use of CR and more use of maladaptive emotion regulation strategies such as rumination. ${ }^{12-14}$ It has been proposed that this habitual pattern in depressed individuals might originate from deficits in the inhibition of negative material which is thought to enhance ruminative thoughts but hampering the effective reappraisal of negative information. ${ }^{15}$ To experimentally investigate CR in MD, studies applied a well-established CR paradigm during which participants are instructed to reappraise negative pictures. This regulation condition is compared with an unregulated condition, during which participants are asked to simply attend to negative stimuli. Within the framework of this paradigm, a number of studies in MD collected behavioural measures of self-reported affect to assess regulation success. Contrary to expectation, some studies found no group differences between depressive and healthy participants in this index of CR. ${ }^{16}{ }^{17}$ However, these results might be biased due to social desirability which probably obscures group differences. Moreover, complex ER disturbances are unlikely to be captured using simple affect ratings, ${ }^{18} 19$ highlighting the importance of additionally assessing objective and more sensitive parameters when studying CR, including neurobiological indices.

A commonly studied neurobiological index of CR is the late positive potential (LPP). Studies in healthy samples show that the LPP is reduced when individuals are instructed to reappraise unpleasant stimuli compared with attending to unpleasant pictures. ${ }^{20-22}$ This decrease is thought to reflect a reduction of the emotional response following CR, which may result from a shift in stimulus meaning. ${ }^{23}$ Depressive psychopathology has been shown to be associated with a smaller reduction of the LPP during CR. ${ }^{23}{ }^{25}{ }^{26}$ However, to date, no study has investigated whether this neurobiological disturbance can be normalised by a repeated training of CR.

Besides the LPP, a number of studies in healthy individuals have investigated gaze fixation patterns to gain insight into the mechanisms underlying CR. ${ }^{27-29}$ They found that a greater regulation success is paralleled by less gaze fixations directed towards negative picture cues. This suggests that the regulatory effects of CR may partly be attributed to gaze deployment, highlighting the importance of also assessing gaze fixation when examining CR.

\section{Trainability of cognitive reappraisal}

Evidence from a randomised controlled trial (RCT) in adult MD suggests that systematically training ER skills in addition to standard CBT improves treatment efficacy. ${ }^{9} 10$ As the ER training of this RCT aimed at enhancing general ER skills and included CR among other ER strategies, the unique contribution of CR remains to be investigated. A recent investigation addressed this issue experimentally in healthy adults, ${ }^{30}$ demonstrating that a task-based CR training resulted in less perceived stress in daily life. Beneficial effects of a task-based CR training have recently also been shown in healthy and anxious adolescents. ${ }^{31}$ While these results are encouraging, it needs to be examined whether the findings can be extended to MD samples and whether a CR training results in reductions in depressive symptomatology, including ruminative thoughts. In this context, it has been proposed that CR training improves cognitive control abilities, including the ability to inhibit negative material. As impairments in the ability to inhibit negative information are thought to play a causal role in rumination, training the ability to reappraise negative information should thus reduce ruminative thoughts. ${ }^{15}$

\section{Aim of the study}

The aim of the study is to assess whether in MD adolescents, a task-based CR training results in an improved downregulation of negative affective responses as assessed by behavioural and neurobiological indices. Moreover, the study aims to investigate whether the training effects generalise outside the laboratory, that is, lead to affectrelated changes and a reduction in perceived stress and rumination.

It is hypothesised that over the course of the CR training, MD adolescents show improvements in the downregulation of negative affective responses as assessed by taskbased behavioural ${ }^{30}$ and neurobiological indices. Based on prior research, ${ }^{30} 31$ it is further hypothesised that the CR training results in improvements in affect-related and stress-related symptoms in daily life.

\section{METHODS AND ANALYSIS}

The study (Public and scientific Title: Cognitive Reappraisal in Adolescents with Major Depression: From Neurobiological Mechanisms to Intervention; German acronym: KONNI) was prospectively registered on ClinicalTrials. gov before recruitment start. The study protocol and the template informed consent/assent forms (including two amendments) were approved by the institutional review board of the local ethics committee (Ethics Committee of the Medical Faculty of the LMU Munich, Germany; study ID: 63-16) on $30^{\text {th }}$ January 2019 and are reported in line with the Standard Protocol Items: Recommendations for Interventional Trials Statement (SPIRIT 2013). Important modifications to the study protocol would 
require an additional amendment, which would have to be approved by the Ethics Committee before implementation. The trial registry (ClinicalTrials.gov) would then be updated.

\section{Design}

This interventional study is designed as a short-term randomised controlled, participant-blind clinical superiority trial including two parallel groups of MD adolescents. Participants will be randomly assigned to a group that receives task-based training in CR or to a control training. All participants will take part in one diagnostic assessment (T0), four training sessions (T1 to T4) over the course of 2 weeks and a follow-up 2 weeks after T4. All questionnaires and test procedures are summarised in table 1. During the training sessions, task-based indices of CR will be continuously assessed based on behavioural affect ratings and the LPP. Moreover, eye gaze will be recorded during the task-based training. The KONNI study design is depicted in figure 1 .

\section{Participants}

Participants aged 12 to 18 years with a current International Classification of Diseases, 10th Revision(ICD-10) diagnosis of $\mathrm{MD},{ }^{32}$ an $\mathrm{IQ}$ of $\geq 80$ and sufficient German language skills will be included. The following exclusion criteria will be applied: acute suicidality, neurological disorders, schizophrenic disorder, pervasive developmental disorder, bipolar disorder, borderline personality disorder, substance dependence disorder and gender dysphoria. Participants with other psychiatric comorbidities are included if MD is the primary diagnosis.

\section{Recruitment}

MD adolescents will be primarily recruited from the Department of Child and Adolescent Psychiatry, Psychosomatics and Psychotherapy, Hospital of the LudwigMaximilians-University (LMU) Munich, Germany. Currently untreated MD patients from the waiting list, and inpatients and outpatients are enrolled in the study. The first participant was enrolled on $27^{\text {th }}$ May 2019. The planned completion of participant enrolment is in April 2021. Since the start of the study $n=35$ subjects were enrolled.

\section{Procedure}

Adolescents and one parent/legal custodian (for participants $<18$ years) will be contacted by an experienced study nurse certified in Good Clinical Practice and will be informed about the study details, including the fact that the allocation to the training groups will be made based on a predefined randomisation list. All potential study participants and their parents/legal custodians will be approached by the study nurse unless it is known beforehand that the exclusion criteria are met (eg, acute suicidality, gender dysphoria). If the clinicians of potential participants conclude that the capacity to provide informed consent/assent are not met (eg, in case of insufficient German skills, cognitive disability or an acute crisis), participants and their parents/legal custodians will not be approached. In case of interest in participation, their written informed consent/assent will be collected. In the written informed consent/assent, participants and their parent/legal custodian are asked for their permission that the data can also be used for an ancillary case-control study on neurobiological underpinnings of ER in MD. The participants and the parent/ legal custodian will be blinded regarding allocation to the $\mathrm{CR} /$ control training group until study completion and only unblinded prior to study completion if they terminate participation prematurely. At the first session (T0), a diagnostic interview and other baseline measures (see below) will be applied to the adolescent and will last $\sim 2.5$ hours. The diagnostic session will be conducted by psychologically trained staff.

If the participant is eligible for the study, the following session appointments will be scheduled and the participant will be randomly allocated to the CR or the control training group. To ensure allocation concealment, the random allocation to one of the groups is implemented after completion of baseline measurements; that is, neither the recruiter nor the person conducting the baseline session know to which group the participant will be assigned after T0. Access to the allocation list is limited to the principal investigator (EG) and her deputy (LF), who will inform the experimenters about the allocation of the participant shortly before the first training session (after the diagnostic session and the decision to include the participant in the study). Randomisation stratifying for age ( $<15$ years vs $\geq 15$ years of age) (based on prior studies in $\mathrm{MD}$ adolescents conducted in our department, 15 is the expected approximate median age of MD participants) and sex will be performed by a statistician, who is neither involved in recruitment nor in testing of participants. The randomisation will be performed with a 1:1 allocation. A follow-up session will take place 2 weeks after completion of the fourth training session. After the follow-up, participants are unblinded regarding group allocation by one of the experimenters. As expense allowance for study participation ( 10 hours including follow-up), the participants will receive $100 €$. Participation is voluntary and can be discontinued at any time for any reason. Participation is discontinued in case of acute suicidality during the study. No other criteria for discontinuation are defined. To assess the feasibility of conducting a large-scale multicentre RCT on the effects of a CR training in addition to standard treatment, the following data will be collected and reported: participation and non-participation rate, drop-outs and reasons for drop-outs, training attendance rates and spontaneously reported adverse effects. Treatment fidelity concerning the training is assured by standardised oral and written instructions and by comprehensive training of the experimenters. The experimenters will be either clinical psychologists or advanced and in-depth trained psychology students. The concomitant treatment as usual is permitted during the ongoing study and information on the type of treatment during 


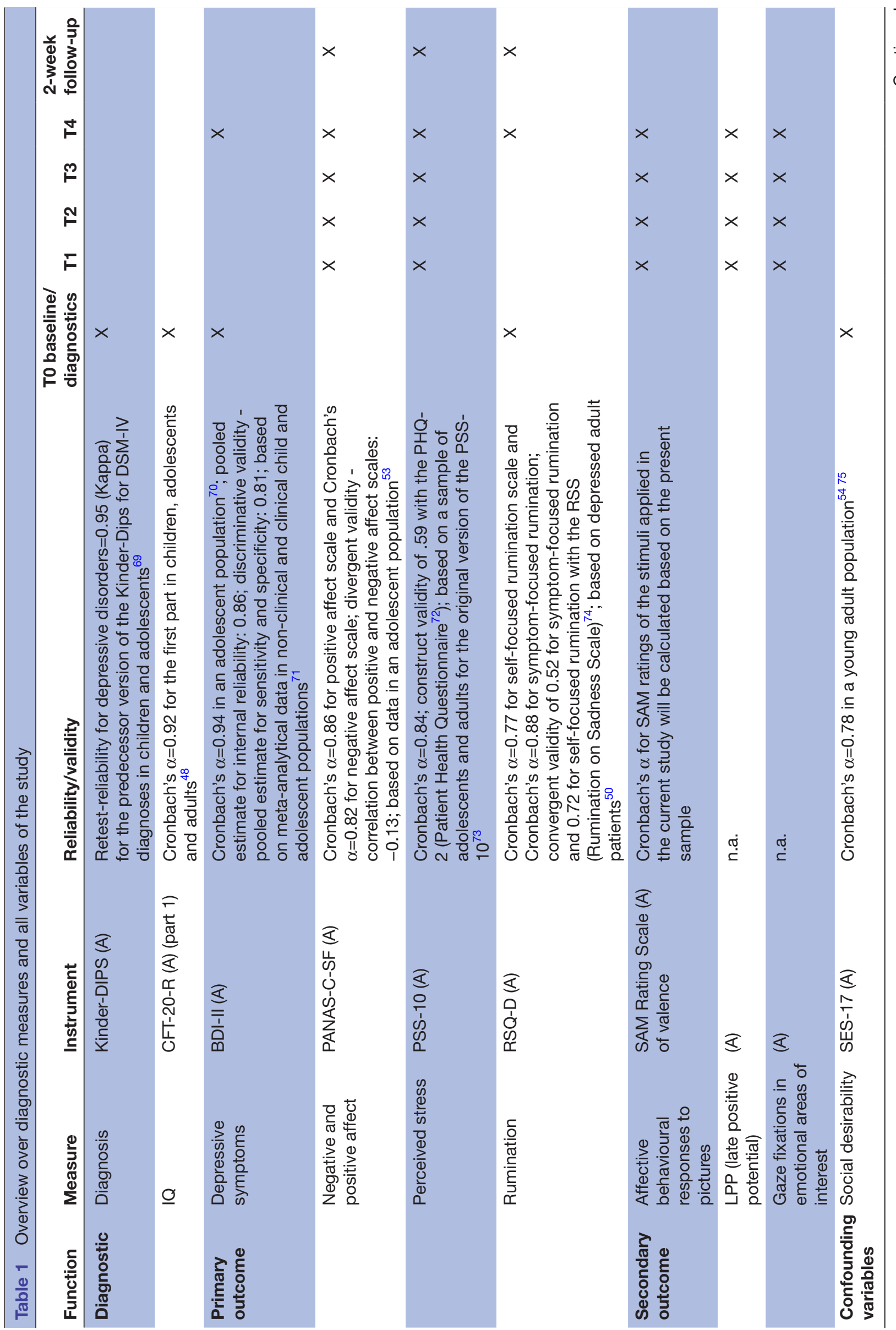




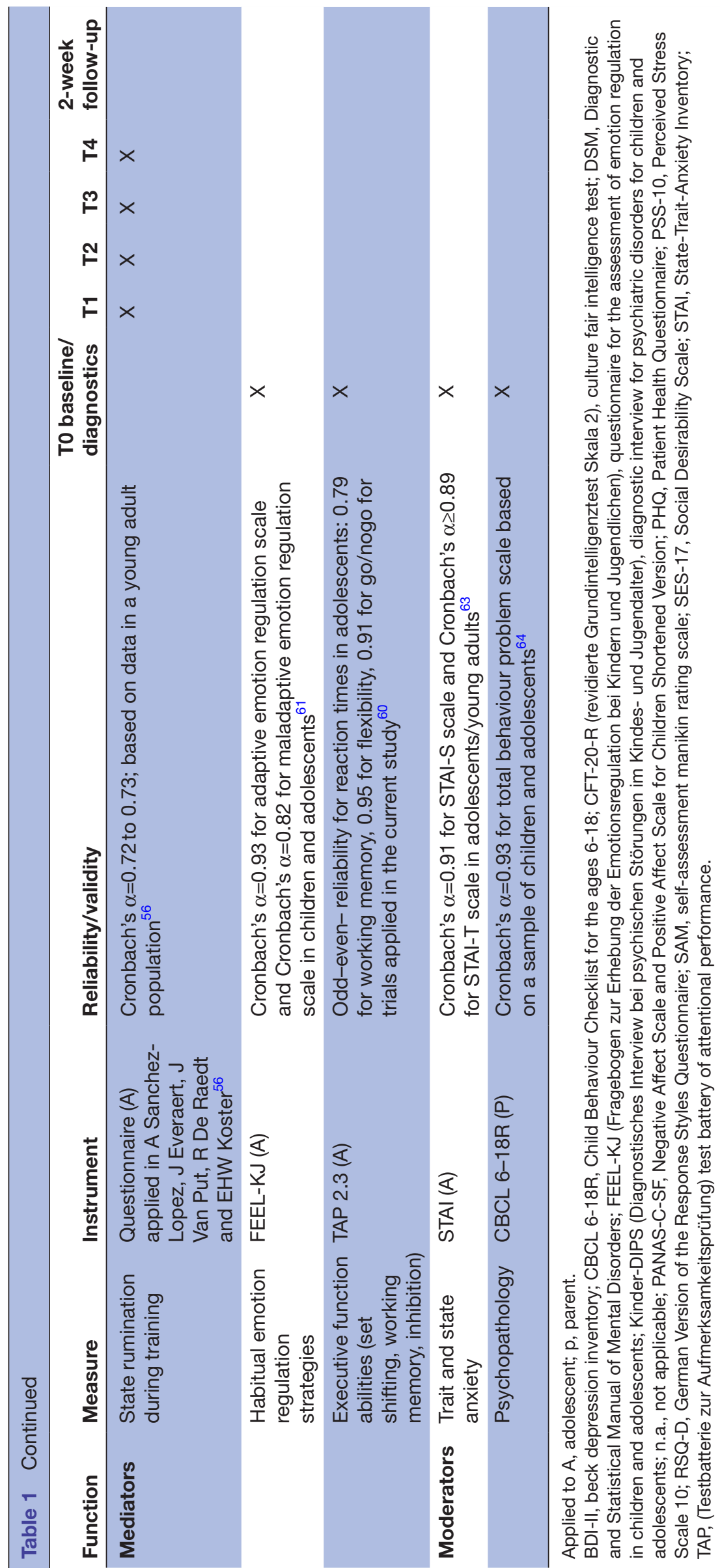




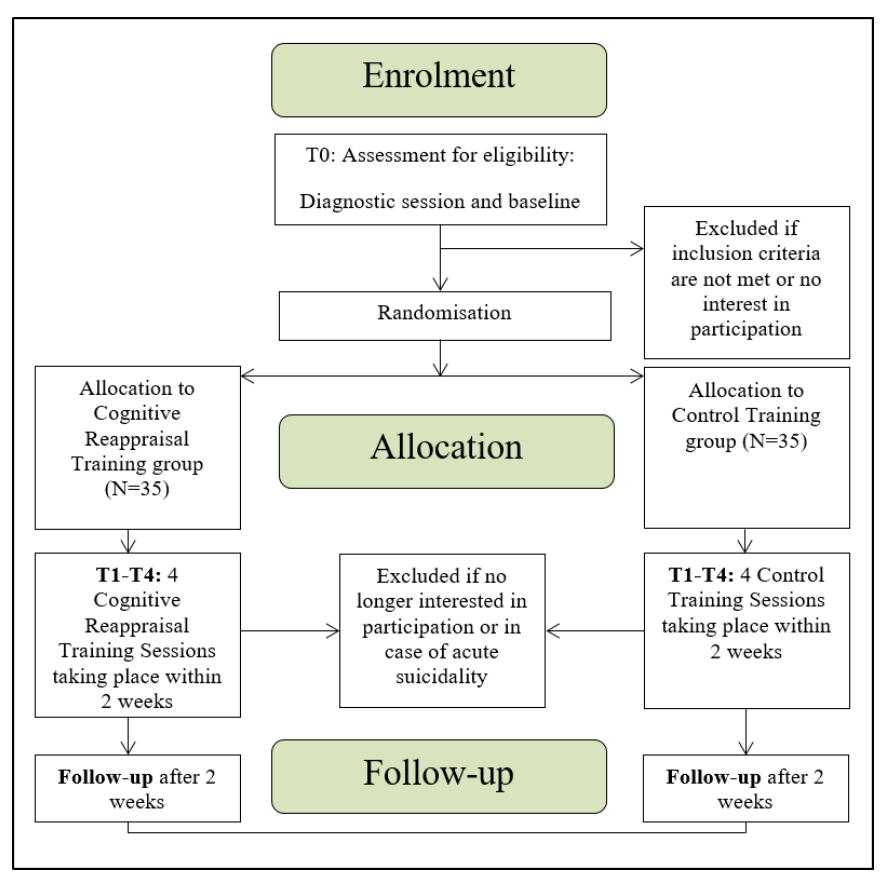

Figure 1 Overview of the KONNI study design.

the study will be assessed. Moreover, patient safety data will be assessed by recording any spontaneously reported adverse effects.

\section{Cognitive reappraisal training}

The CR training task is well-established and is adapted from previous studies, ${ }^{22}{ }^{33-35}$ including a study from our group, in which we demonstrated that adolescents with MD understand and comply with task instructions, and are able to downregulate negative affective responses to negative pictures via CR. ${ }^{36}$ The training procedure was adapted from a previous study. ${ }^{30}$ An exemplary illustration of an experimental trial is depicted in figure 2. Participants will be presented emotional pictures which are preceded by a condition specific cue signalling the appropriate strategy (attend vs reappraise) during

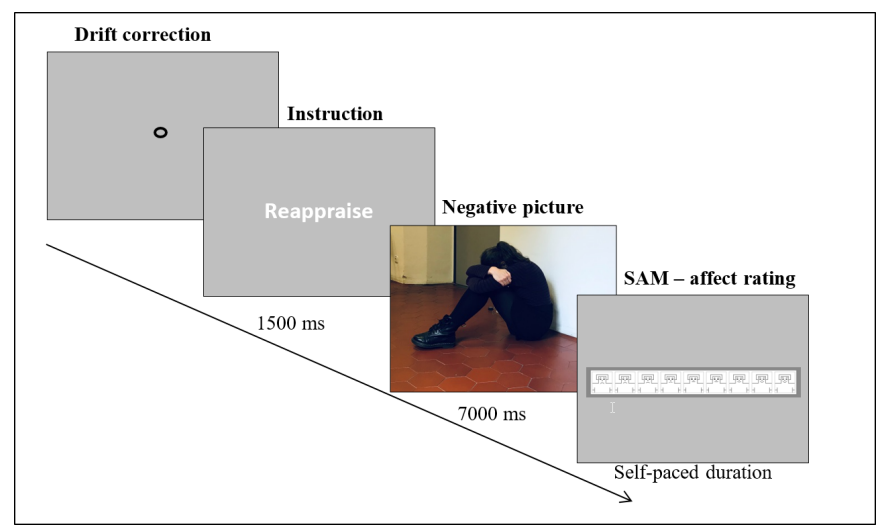

Figure 2 Experimental time course. Exemplary illustration of a trial belonging to the $\mathrm{CR}$ training (negative-reappraise condition). The picture shown is exemplary and not part of the picture databases used in the study. CR, cognitive reappraisal; SAM, self-assessment manikin rating scale (as a portrait version). picture presentation. Following each picture, participants are instructed to indicate their affective response to the image on the portrait version of the 9-point selfassessment manikin scale for valance $\left(\mathrm{SAM}^{37}\right.$; for the portrait version $\mathrm{see}^{38}{ }^{39}$ ), which has been frequently applied in youth samples. ${ }^{40}{ }^{41}$ A higher score on this scale indicates a more positive valence rating. The task involves four conditions: (1) Negative-reappraise: participants are asked to view negative pictures and to decrease their affective response by reappraising the negative event. Participants are explicitly instructed to use the reinterpretation tactic during CR, which involves 'mentally changing the meaning of the actions, context and/or outcome depicted in a stimulus'.$^{30}$ (2) Negative-attend, (3) Neutral-attend and (4) Positive-attend: participants are asked to view the pictures and to respond naturally to them without trying to alter their affective response. Comparison of the negative-reappraise and the negativeattend conditions allows assessing behavioural and neural indices of reappraisal success. The neutral-attend and the positive-attend conditions are included to avoid slight changes in negative affect due to the exclusive presentation of unpleasant pictures. ${ }^{42}$

The training task will be repeated over four training sessions. Before the first session, participants will complete a comprehensive practice training, during which a trained experimenter will provide standardised oral instructions regarding the task and the two strategies (attend and reappraise). ${ }^{30} 36$ Thereafter, walk-through images are presented to confirm the appropriate use of the strategies. Task instructions will be briefly summarised before each training session.

In each training session, 144 pictures will be presented in three blocks, each containing 12 positive-attend, 12 neutral-attend, 12 negative-attend and 12 negativereappraise trials. Developmentally appropriate pictures (eg, excluding pictures of dead persons or erotic images) are taken from the International Affective Picture System $\left(\right.$ IAPS $^{43}$ ) Besançon Affective Picture Set-Adolescents (BAPS-Ado ${ }^{44}$ ) and Besançon Affective Picture Set-Adults (BAPS-Adult ${ }^{45}$ ) with the latter two sets being derived from the Besançon Attachment Pictures Set. Each picture will be presented twice over the course of the training. ${ }^{30}$ To ensure adherence to task instructions, participants will fill in a questionnaire after each training session to indicate which strategies they used during the task.

\section{Control training}

The control training is implemented to account for unspecific effects and the task employed is similar to the CR task except that it involves no 'negative-reappraise' condition. Instead, the control task only involves attend conditions (negative-attend, neutral-attend and positiveattend). To keep the total number of trials involving negative pictures constant across the two groups, the control task involves twice as many negative-attend trials as the CR training group. Mirroring the CR training procedure, the control training is repeated over four sessions and 
preceded by standardised oral instructions and a practice session. Participants of the control training group will also fill in a questionnaire on how they resolved the task after each training session.

\section{MEASURES}

Information about psychometric properties of all measures described in the following paragraph are summarised in table 1 along with the respective assessments points.

\section{Diagnostic measures}

A diagnosis of MD and potential comorbidities will be assessed using a well-established German standardised semi-structured diagnostic interview (Kinder-DIPS). ${ }^{46} 47$ The interview will be administered by experienced, psychologically trained experimenters. To assess inter-rater reliability based on Cohen's kappa (k), 10\% of the KinderDIPS interviews will be rated by two experimenters. IQ will be estimated based on the first part of the CFT-20-R. ${ }^{48}$ For some patients, results from other established IQ tests $\left(\mathrm{eg}\right.$, WISC-V $\left.{ }^{49}\right)$ will be available from routine care, which will be used instead.

\section{Outcome measures}

\section{Primary outcomes}

The primary outcomes of the study are (1) change in rumination from baseline (T0) to post-training (directly after T4) and follow-up (assessed by the scales 'selffocused rumination' and 'symptom-focused rumination' of the German short version of the Response Styles Questionnaire; RSQ-D ${ }^{50}{ }^{5}$; (2) change in depressive symptoms (assessed by the German version of the Beck Depression Inventory - Second Edition; BDI-II ${ }^{51}$ ) from baseline (T0) to post-training (directly after T4); (3) change in perceived stress over the course of the four training sessions and at follow-up (assessed by the German translation of the Perceived Stress Scale 10; PSS-10; by Professor Dr Arndt Büssing ${ }^{52}$ ) (in the PSS-10, items were reworded to 'in the past few days' instead of 'in general' to better allow assessing changes of the course of the training); (4) changes in negative and positive affect (assessed by the Negative Affect Scale and Positive Affect Scale for Children Shortened Version; PANAS-C-SF ${ }^{53}$ ) over the course of the four training sessions and at follow-up.

\section{Secondary outcomes}

One secondary outcome is the change in the downregulation of affective behavioural responses to negative pictures by means of CR. Affective behavioural responses to negative pictures will be continuously assessed during each of the four training sessions using the SAM rating scale. ${ }^{37}$ (with the portrait version from ${ }^{38}{ }^{39}$ )

Another secondary outcome is the change in the downregulation of the early and late LPP amplitude to negative pictures by means of CR. The LPP elicited to pictures in the negative-attend and the negative-reappraise condition will be continuously assessed during each of the four training sessions. Additionally, changes in the percent duration of gaze fixations within a-priori defined emotional interest areas of negative pictures (emotional areas of interest of negative pictures will be defined based on a separate validation study) will be assessed during both the negative-attend and negative-reappraise condition over the course of the four training sessions.

All secondary outcome measures will only be assessed in the CR training group, as participants in the control training group are not instructed to apply CR. However, these measures will also be assessed in the control training group during the negative-attend condition to allow additional exploratory analyses.

\section{Confounding variable}

Participants will complete the Social Desirability Scale (SES-17) ${ }^{54}$ The rationale for assessing this confounding variable is that behavioural ratings of affective responses to pictures will be assessed, which are prone to response biases. $^{18}$

\section{Mediators}

State rumination during the training will be treated as a potential mediator as ruminative thoughts might hamper beneficial training effects. ${ }^{55}$ After completion of each training session, participants in the CR and control training group rate state rumination during the task on a scale from 0 'not at all' to 10 'exactly' based on a 5-item questionnaire applied in a previous study. ${ }^{56}$

We will also assess a potential mediating role of executive function abilities, which are thought to be critically involved in ER. ${ }^{57}$ Individual baseline differences in executive function abilities might thus prove important mediators of training outcome. Therefore, set-shifting, working memory and inhibition ${ }^{58}{ }^{59}$ as the three core components of executive functions will be assessed based on the TAP 2.3 (Testbatterie zur Aufmerksamkeitspruefung). ${ }^{60}$

Moreover, we will treat habitual ER as a potential mediator, which will be assessed based on the FEEL-KJ. ${ }^{61}$ The FEEL-KJ is a self-report questionnaire assessing habitual adaptive and maladaptive ER strategies.

\section{Moderators}

As anxiety disorders/symptoms frequently co-occur with $\mathrm{MD},{ }^{62}$ state and trait anxiety will be assessed based on the State-Trait-Anxiety Inventory (STAI) ${ }^{63}$ Moreover, symptoms of psychopathology will be screened using the Child Behaviour Checklist (CBCL 6-18R) ${ }^{64}$ Both variables will be treated as potential moderators.

\section{Eye-tracking apparatus}

During the four training sessions, eye-movements will be continuously recorded using the Eyelink 1000 Plus (SR Research Ltd.) eye-tracker. At the beginning of the training, participants are seated at the viewing distance of approximately $55 \mathrm{~cm}$ from the monitor and a 9-point calibration is performed. Prior to each experimental trial, a drift correction is conducted. 


\section{Electroencephalogram recording and preprocessing}

The electroencephalogram data is recorded using the Electrical Geodesics, Inc, 128-channel system with $500 \mathrm{~Hz}$ sampling rate and $\mathrm{Cz}$ as reference electrode. Impedance is kept below $50 \mathrm{k} \Omega$ during recording. The further preprocessing and analyses steps will be performed with Brainvision Analyzer 2.1 (Brain Products GmbH, Germany). After filtering, removal of electro-oculographic artefacts based on Independent Component Analysis and exclusion of other artefacts, the signal will be re-referenced. Data will be segmented into epochs, baseline-corrected and averaged separately for each participant and condition. The LPP will be measured as the mean amplitude at a centro-parietal region-of-interest within early $(<300$ $\mathrm{ms}$ ) and late time windows ( $\geq 300 \mathrm{~ms}$ ) following picture onset. $^{3365}$

\section{Data management and confidentiality}

All data will be entered electronically in IBM SPSS statistics by a scientific assistant. Plausibility and completeness checks will be regularly performed by EG, LF and CP to promote and monitor data quality. Original documents will be maintained for 15 years in the department. All original and electronic data is stored on the hospitals server under a pseudorandomised coded ID, which does not contain names or birth dates of the participants. Only the project leader and her deputies have access to the participants' names and the corresponding allocation to the encryption code and the original documents, which are kept in locked cabinets. The records containing names and other personal identifiers (eg, informed consent/assent forms) will be stored in locked cabinets separately from the study records which are identified by the coded ID. The participants' study information will not be released outside the study, except in case of threat to self or threat to others. The final cleaned data sets will be provided to all investigators of the study. To adhere to principles of open science and to facilitate further use of aggregated data in meta-analytical approaches, we will consider making raw data available to other researchers if this can be achieved along with protecting sensitive patient information, such as sociodemographic information. Since patients could possibly be identified by making our raw data publicly available, ethical principles of protecting patient confidentiality would be breached. Aggregated group data can be made available on request.

\section{Calculation of sample size}

A rough approximation of the requested sample size targeted at rumination as one of the primary outcomes can be achieved by referring to a previous study. ${ }^{66}$ This study investigated the effects of a task-based cognitive control training compared with treatment as usual in adult MD. The training aimed at improving ER skills indirectly by targeting mechanisms thought to underlie ER. This study reported a large effect size in the training group from pre to post (Cohen's $\mathrm{d}=1.42$ ) on a measure related to negative affect (rumination). Based on a conservative assumption, a large effect size of $\mathrm{d}=0.85$ is expected with regard to changes in rumination for the CR training from pre to post. For the control training, a small effect size $(d=0.2)$ is expected from pre to post due to unspecific 'positive' effects of the control intervention. This effect size is based on prior evidence (detailed statistical information was derived from personal communication with corresponding author of this study; B Denny, $26^{\text {th }}$ April 2017) on the effects of a similar control training in young adults. $^{30}$

Based on these assumptions, calculation of the required sample size to detect a significant 2 (group) x 2 (time: $\mathrm{T} 0=$ pre, $\mathrm{T} 4=$ post $)$ interaction for rumination using a repeated measures analysis of variance (ANOVA) with a $5 \%$ level of significance (alpha $=0.05$ ) and $80 \%$ statistical power (1-beta $=0.80$ ) revealed an $\mathrm{N}=47$ for the total group (assuming a 0.7 pre-post correlation). The drop-out rate for the study is estimated at approximately 33\%. Thus, $\mathrm{n}=35$ adolescents will be enrolled in each group. The necessary sample size was calculated with $G^{*}$ power 3.1. Statistical power of analyses that involve more measurements, such as the analysis of affect, will have more than $80 \%$ statistical power if effect sizes are similar.

\section{Statistical analysis}

To test the hypotheses that the CR training results in a stronger decrease in depressive symptoms/rumination from baseline to post-training assessment compared with a control training, a mixed-model ANOVA with the factors group (CR vs control training) and time (T0 and T4) will be conducted for the BDI-II and the two RSQ-D scales 'self-focused rumination' and 'symptom-focused rumination'. To account for the fact that the RSQ-D will be reassessed at follow-up, a mixed-model ANOVA with the factors group (CR vs control training) and time (T0, T4 and follow-up) will additionally be conducted for the two rumination scales. Mixed modelling (also known as multilevel analysis, with observations 'nested' within participants) has the advantage over regular repeated measures ANOVA that all available data can be used, including data from incomplete cases, without using imputation techniques for missing data. ${ }^{67}$ To test the hypotheses that the CR training results in a (1) stronger decrease in negative and (2) stronger increase in positive affect from pre-training to post-training and follow-up assessment compared with the control training, mixedmodel ANOVAs with the factors group (CR vs control training) and time (T1, T2, T3, T4 and follow-up) will be conducted for the Negative Affect/Positive Affect scale of the PANAS-C-SF. The same statistical model procedure will be applied for perceived stress (PSS-10).

To test the hypothesis that over the course of the CR training, MD adolescents show an increased downregulation of the early and late LPP amplitude via CR, a mixed-model ANOVA with the factors 2 (condition: negative-reappraise and negative-attend) $\mathrm{x} 4$ (training session: T1, T2, T3 and T4) will be conducted separately for early and late LPP time windows. The same statistical 
procedure will be conducted for the affective ratings of negative pictures and the percent of gaze fixation duration in emotional areas of negative pictures. Correlation analyses will be performed between the percent of gaze fixation duration in emotional areas of negative pictures and ER success (downregulation of the LPP and affective ratings of negative pictures via CR).

To examine changes of the LPP, affective ratings of negative pictures and gaze fixations for the negativeattend condition in the control training group over the course of the training, additional exploratory ANOVAs will be conducted. Likewise, for the control training group, exploratory correlation analyses will be calculated to examine relationships between these measures and gaze fixation duration in emotional areas of negative pictures. Finally, moderation and mediation analyses will be applied to examine the role of mediating and moderating variables.

\section{Patient and public involvement}

Participants or their parents were not involved in designing the study. However, we will present the results of the study to parent and participant representatives to include their suggestions in the dissemination plan. Moreover, parallel to publication of the results, all participants and their families will receive a letter summarising the main findings and conclusions in comprehensible language.

\section{DISCUSSION}

This study will for the first time elucidate whether in adolescents with MD, a CR training shows beneficial effects both in and outside the laboratory. If the training proves effective, this approach might be a promising resource-effective intervention for adolescents with MD. Such an intervention could, for example, be applied to bridge the often long waiting times for treatment of $\mathrm{MD}$ or as an adjunction to a treatment as usual. Moreover, after psychoeducation on CR and guided practice of this strategy in psychotherapy, the training could also be (online) applied as an adjunct to treatment as usual. In such a context, the psychotherapist could monitor difficulties and progress in the use of $\mathrm{CR}$, and promote the transfer of strategy into everyday life of the patient.

Building on the results from the present RCT, future investigations could additionally consider transfer effects to CR tasks other than the training tasks or to CR abilities in daily life, for example, based on ecological momentary assessment. Moreover, as a next step, it would be worthwhile to assess whether this training might prove effective in patients with other psychiatric disorders which are also characterised by deficient ER abilities, such as anxiety disorders and eating disorders. ${ }^{68}$

A limiting factor of the study is the short follow-up interval of 2 weeks. Thus, future studies should include a longer follow-up interval to also examine whether the effects of the training are long-lasting. Another limitation is that the study is single-blinded (participant-blinded) concerning the allocation to the CR training versus control training. This single-blinding procedure entails the risk that the experimenters will transfer their expectations to the participants. However, as the participants will perform a comprehensive practice training that is guided by the experimenter, double-blinding would not be feasible. Finally, it should be stated that the present study does not include the ecological momentary assessment of outcome measures. Expanding on the present study, it would be important to also apply experience sampling methods in future work to be able to draw comprehensive conclusions regarding transfer effects of the CR training to daily live. Despite these caveats, the results of our study will be an important step towards larger scale, multicentre RCTs in MD adolescents to investigate whether a CR training increases the efficacy of standard treatments. Furthermore, the study will elucidate neurobiological changes that occur during training and whether these are linked to changes in training outcome. To this end, our protocol may also aid to identify potential biomarkers for monitoring and predicting treatment success and thereby spark further research into the direction of individualised treatment adaptation based on neurobiological parameters.

\section{Ethics and dissemination}

Written informed consent/assent will be taken by the participating adolescents and their parents/legal custodians. The study protocol and the template informed consent/assent forms were approved (including two amendments) by the institutional review board of the local ethics committee (Ethics Committee of the Medical Faculty of the LMU Munich, Germany; study ID: 63-16) on $30^{\text {th }}$ January 2019.

Study results will be presented at national and international conferences and published in peer-reviewed publications. Moreover, the participants and their parents will receive a summary of the study results in layman's language.

\section{Author affiliations}

${ }^{1}$ Department of Child and Adolescent Psychiatry, Psychosomatics and Psychotherapy, Hospital of the Ludwig-Maximilians-University (LMU) Munich, Munich, Germany

${ }^{2}$ Research Institute of Child Development and Education, University of Amsterdam, Amsterdam, Netherlands

${ }^{3}$ Translational Brain Medicine in Psychiatry and Neurology, Department of Child and Adolescent Psychiatry, Psychosomatics, and Psychotherapy, RWTH Aachen University, Aachen, Germany

${ }^{4}$ JARA-Brain, Aachen, Germany

${ }^{5}$ Department of Child and Adolescent Psychiatry and Psychotherapy, University Medical Center Göttingen, Göttingen, Germany

Acknowledgements We are grateful to Carolin Zsigo for the assistance during the implementation of the experiment. We wish to thank Petra Wagenbüchler for her assistance in data collection. We further would like to thank Dr Lauriane Vulliez and Dr Monika Szymanska for providing BAPS-Ado and BAPS-Adult picture sets.

Contributors EG, LF, GSK and CP contributed to the study design. Data management will be conducted by $L F$ and $E G$ and data analysis and interpretation will be performed by LF, EG, FO, GSK, JB, MSR and CP. EG and LF wrote the study 
protocol. All authors read and critically revised the draft of the paper and approved the final version of this manuscript.

Funding This work was supported by the Deutsche Forschungsgemeinschaft (DFG, German Research Foundation) - Grant GR4753/2-1 to EG. The funding body played no role in the design of the study or writing of the manuscript or the decision to submit the report for publication.

Competing interests None declared.

Patient and public involvement Patients and/or the public were involved in the design, or conduct, or reporting, or dissemination plans of this research. Refer to the Methods section for further details.

Patient consent for publication Not required.

Provenance and peer review Not commissioned; externally peer reviewed.

Open access This is an open access article distributed in accordance with the Creative Commons Attribution Non Commercial (CC BY-NC 4.0) license, which permits others to distribute, remix, adapt, build upon this work non-commercially, and license their derivative works on different terms, provided the original work is properly cited, appropriate credit is given, any changes made indicated, and the use is non-commercial. See: http://creativecommons.org/licenses/by-nc/4.0/.

\section{ORCID iDs}

Ellen Greimel http://orcid.org/0000-0002-9916-9230

Martin Schulte-Rüther http://orcid.org/0000-0002-7198-9923

\section{REFERENCES}

1 Kessler RC, Bromet EJ. The epidemiology of depression across cultures. Annu Rev Public Health 2013;34:119-38.

2 Avenevoli S, Knight E, Kessler RC, et al. Epidemiology of depression in children and adolescents. In: Abela JRZ, Hankin BL, eds. Handbook of depression in children and adolescents. New York: Guilford, 2008: 6-32.

3 Kovacs M, Yaroslavsky I. Practitioner review: dysphoria and its regulation in child and adolescent depression. J Child Psychol Psychiatry 2014;55:741-57.

4 Ochsner KN, Gross JJ. The cognitive control of emotion. Trends Cogn Sci 2005;9:242-9.

5 Ahmed SP, Bittencourt-Hewitt A, Sebastian CL. Neurocognitive bases of emotion regulation development in adolescence. Dev Cogn Neurosci 2015;15:11-25.

6 Compton SN, March JS, Brent D, et al. Cognitive-Behavioral psychotherapy for anxiety and depressive disorders in children and adolescents: an evidence-based medicine review. J Am Acad Child Adolesc Psychiatry 2004:43:930-59.

7 Curry J, Rohde P, Simons A, et al. Predictors and moderators of acute outcome in the treatment for adolescents with depression study (TADS). J Am Acad Child Adolesc Psychiatry 2006;45:1427-39.

8 Thapar A, Collishaw S, Pine DS, et al. Depression in adolescence. Lancet 2012:379:1056-67.

9 Berking M, Ebert D, Cuijpers $\mathrm{P}$, et al. Emotion regulation skills training enhances the efficacy of inpatient cognitive behavioral therapy for major depressive disorder: a randomized controlled trial. Psychother Psychosom 2013;82:234-45.

10 Berking M, Wupperman P, Reichardt A, et al. Emotion-regulation skills as a treatment target in psychotherapy. Behav Res Ther 2008;46:1230-7

11 Cutuli D. Cognitive reappraisal and expressive suppression strategies role in the emotion regulation: an overview on their modulatory effects and neural correlates. Front Syst Neurosci 2014;8:175

12 Garnefski N, Kraaij V. The cognitive emotion regulation questionnaire: psychometric features and prospective relationships with depression and anxiety in adults. Eur J Psychol Assess 2007;23:141-9.

13 Joormann J, Gotlib IH. Emotion regulation in depression: relation to cognitive inhibition. Cogn Emot 2010;24:281-98.

14 Schäfer Johanna Özlem, Naumann E, Holmes EA, et al. Emotion regulation strategies in depressive and anxiety symptoms in youth: a meta-analytic review. J Youth Adolesc 2017;46:261-76.

15 Cohen N, Daches S, Mor N, et al. Inhibition of negative content-a shared process in rumination and reappraisal. Front Psychol 2014:5:622.

16 Dillon DG, Pizzagalli DA. Evidence of successful modulation of brain activation and subjective experience during reappraisal of negative emotion in unmedicated depression. Psychiatry Res 2013;212:99-107.
17 Sheline YI, Barch DM, Price JL, et al. The default mode network and self-referential processes in depression. Proc Natl Acad Sci U S A 2009;106:1942-7.

18 Zilverstand A, Parvaz MA, Goldstein RZ. Neuroimaging cognitive reappraisal in clinical populations to define neural targets for enhancing emotion regulation. A systematic review. Neuroimage 2017;151:105-16.

19 Greimel E, Schulte-Rüther M, Fink GR, et al. Development of neura correlates of empathy from childhood to early adulthood: an fMR study in boys and adult men. J Neural Transm 2010;117:781-91.

20 DeCicco JM, O'Toole LJ, Dennis TA. The late positive potential as a neural signature for cognitive reappraisal in children. Dev Neuropsychol 2014;39:497-515.

21 Moser JS, Krompinger JW, Dietz J, et al. Electrophysiological correlates of decreasing and increasing emotional responses to unpleasant pictures. Psychophysiology 2009;46:17-27.

22 Paul S, Simon D, Kniesche R, et al. Timing effects of antecedentand response-focused emotion regulation strategies. Biol Psychol 2013;94:136-42.

23 Dennis TA, Hajcak G. The late positive potential: a neurophysiological marker for emotion regulation in children. J Child Psychol Psychiatry 2009;50:1373-83.

24 Wessing I, Rehbein MA, Romer G, et al. Cognitive emotion regulation in children: reappraisal of emotional faces modulates neural source activity in a frontoparietal network. Dev Cogn Neurosci 2015;13:1-10.

25 Bylsma LM. Emotional Reactivity and Regulation in Current and Remitted Depression: An Event Related Potential Study [Dissertation] University of South Florida; 2012.

26 Kudinova AY, Owens M, Burkhouse KL, et al. Differences in emotion modulation using cognitive reappraisal in individuals with and without suicidal ideation: an Erp study. Cogn Emot 2016;30:999-1007.

27 Bebko GM, Franconeri SL, Ochsner KN, et al. Attentional deployment is not necessary for successful emotion regulation via cognitive reappraisal or expressive suppression. Emotion 2014;14:504-12.

28 Manera V, Samson AC, Pehrs C, et al. The eyes have it: the role of attention in cognitive reappraisal of social stimuli. Emotion 2014;14:833-9.

29 Strauss GP, Ossenfort KL, Whearty KM. Reappraisal and distraction emotion regulation strategies are associated with distinct patterns of visual attention and differing levels of cognitive demand. PLoS One 2016;11:e0162290.

30 Denny BT, Ochsner KN. Behavioral effects of longitudinal training in cognitive reappraisal. Emotion 2014;14:425-33.

31 De Witte NAJ, Sütterlin S, Braet C, et al. Psychophysiological correlates of emotion regulation training in adolescent anxiety: evidence from the novel PIER task. J Affect Disord 2017;214:89-96.

32 World Health Organization. The ICD-10 classification of mental and behavioural disorders: clinical descriptions and diagnostic guidelines. Geneva World Health Organization; 1992

33 Schönfelder S, Kanske P, Heissler J, et al. Time course of emotionrelated responding during distraction and reappraisal. Soc Cogn Affect Neurosci 2014;9:1310-9.

34 Paul S, Simon D, Endrass T, et al. Altered emotion regulation in obsessive-compulsive disorder as evidenced by the late positive potential. Psychol Med 2016;46:137-47.

35 Sarlo M, Ubel S, Leutgeb V, et al. Cognitive reappraisal fails when attempting to reduce the appetitive value of food: an ERP study. Biol Psychol 2013;94:507-12.

36 Greimel E, Piechaczek C, Schulte-Rüther M, et al. The role of attentional deployment during distancing in adolescents with major depression. Behav Res Ther 2020;126:103554.

37 Bradley MM, Lang PJ. Measuring emotion: the self-assessment manikin and the semantic differential. J Behav Ther Exp Psychiatry 1994;25:49-59.

38 Suk H-J. Color and Emotion-a study on the affective judgment across media and in relation to visual stimuli [Dissertation] Universität Mannheim; 2006

39 Lang PJ. Behavioral treatment and bio-behavioral assessment: Computer applications. In: Sidowski JB, Johnson JH, Williams TA, eds. Technology in mental health care delivery systems. Norwood, NJ: Ablex, 1980: 119-37.

40 Musser ED, Galloway-Long HS, Frick PJ, et al. Emotion regulation and heterogeneity in attention-deficit/hyperactivity disorder. J Am Acad Child Adolesc Psychiatry 2013;52:163-71.

41 Reichel VA, Schneider N, Grünewald B, et al. "Glass fairies" and "bone children": adolescents and young adults with anorexia nervosa show positive reactions towards extremely emaciated body pictures measured by the startle reflex paradigm. Psychophysiology 2014;51:168-77. 
42 Patrick CJ, Lavoro SA. Ratings of emotional response to pictorial stimuli: positive and negative affect dimensions. Motiv Emot 1997;21:297-321.

43 Lang PJ, Bradley MM, Cuthbert BN. International affective picture system (IAPS): affective ratings of pictures and instruction manual. technical report A-8. Gainesville, FL: University of Florida, 2008.

44 Szymanska M, Monnin J, Noiret N, et al. The Besançon affective picture Set-Adolescents (the BAPS-Ado): development and validation. Psychiatry Res 2015;228:576-84.

45 Szymanska M, Comte A, Tio G, et al. The Besançon affective picture set-adult (BAPS-Adult): development and validation. Psychiatry Res 2019;271:31-8.

46 Margraf J, Cwik JC, Pflug V, et al. Structured clinical interviews for mental disorders across the lifespan: psychometric quality and further developments of the dips open access interviews. Z Klin Psychol Psychopathol Psychother 2017;46:176-86.

47 Margraf J, Cwik JC, Suppiger A, Schneider S. DIPS Open Access: Diagnostic Interview for Mental Disorders. [DIPS Open Access: Diagnostisches Interview bei psychischen Störungen]. Bochum Mental Health Research and Treament Center; 2017.

48 Weiß RH. CFT 20-R, Grundintelligenztest Skala 2 - Revision (2. Auflage) 2019.

49 Wechsler D. Wechsler intelligence scale for Children-Fifth edition (WISC-V. Frankfurt/Main, Nürnberg, Leipzig: Pearson, 2017.

50 Kühner C, Huffziger S, Nolen-Hoeksema S. Response styles Questionnaire-Deutsche version: RSQ-D. Hogrefe, 2007.

51 Hautzinger M, Keller F, Kühner C. Das Beck Depressionsinventar II. Deutsche Bearbeitung und Handbuch zum BDI-II. Frankfurt am Main: Harcourt Test Services, 2006.

52 Cohen S, Kamarck T, Mermelstein R. A global measure of perceived stress. J Health Soc Behav 1983;24:385-96.

53 Ebesutani C, Regan J, Smith A, et al. The 10-Item positive and negative affect schedule for children, child and parent shortened versions: application of item response theory for more efficient assessment. J Psychopathol Behav Assess 2012;34:191-203.

54 Stöber J. Die Soziale-Erwünschtheits-Skala-17 (SES-17): Entwicklung und erste Befunde zu Reliabilität und Validität. Diagnostica 1999;45:173-7.

55 Koster EHW, De Lissnyder E, Derakshan N, et al. Understanding depressive rumination from a cognitive science perspective: the impaired disengagement hypothesis. Clin Psychol Rev 2011;31:138-45.

56 Sanchez-Lopez A, Everaert J, Van Put J, et al. Eye-gaze contingent attention training (ECAT): examining the causal role of attention regulation in reappraisal and rumination. Biol Psychol 2019;142:116-25.

57 Cohen N, Ochsner KN. From surviving to thriving in the face of threats: the emerging science of emotion regulation training. Curr Opin Behav Sci 2018;24:143-55.

58 Miyake A, Friedman NP, Emerson MJ, et al. The unity and diversity of executive functions and their contributions to complex "frontal lobe" tasks: A latent variable analysis. Cogn Psychol 2000:41:49-100.

59 Friedman NP, Miyake A, Young SE, et al. Individual differences in executive functions are almost entirely genetic in origin. $J$ Exp Psychol Gen 2008;137:201-25.
60 Zimmermann P, Fimm B. TAP Testbatterie Zur Aufmerksamkeitsprüfung version 2.3: vera Fimm. Psychologische Testsysteme, 2012

61 Grob A, Smolenski C. Fragebogen Zur Erhebung Der Emotionsregulation bei Kindern und Jugendlichen (FEEL-KJ). 2. Aktualisierte und ergänzte Auflage. Bern: Huber Verlag, 2009.

62 Axelson DA, Birmaher B. Relation between anxiety and depressive disorders in childhood and adolescence. Depress Anxiety 2001;14:67-78.

63 Laux L, Glanzmann P, Schaffner P, et al. Das State-TraitAngstinventar Weinheim. Germany: Beltz, 1981.

64 Döpfner M, Achenbach TM, Plück J. CBCL/6-18R-TRF/6-18RYSR/11-18R: Deutsche Schulalter-Formen Der child behavior checklist von Thomas M. Achenbach: Elternfragebogen über das Verhalten von Kindern und Jugendlichen (CBCL/6$18 R$ ), Lehrerfragebogen über das Verhalten von Kindern und Jugendlichen (TRF/6-18R), Fragebogen für Jugendliche (YSR/1118R. Göttingen: Hogrefe, 2014.

65 Moran TP, Jendrusina AA, Moser JS. The psychometric properties of the late positive potential during emotion processing and regulation. Brain Res 2013;1516:66-75.

66 Siegle GJ, Price RB, Jones NP, et al. You gotta work at it: Pupillary indices of task focus are prognostic for response to a neurocognitive intervention for rumination in depression. Clin Psychol Sci 2014;2:455-71.

67 Murrar S, Brauer M. Mixed model analysis of variance. In: Frey BB ed. The SAGE encyclopedia of educational research, measurement, and evaluation. Thousand Oaks: SAGE Publications, Inc, 2018: p. 1075-8.

68 Aldao A, Nolen-Hoeksema S, Schweizer S. Emotion-regulation strategies across psychopathology: a meta-analytic review. Clin Psychol Rev 2010;30:217-37.

69 Adornetto C, In-Albon T, Schneider S. Diagnostik Im Kindesund Jugendalter anhand strukturierter interviews: Anwendung und Durchführung des Kinder-DIPS. Klin Diagnostik u Evaluation 2008;1:363-77.

70 Dolle K, Schulte-Körne G, O'Leary AM, et al. The Beck depression inventory-II in adolescent mental health patients: cut-off scores for detecting depression and rating severity. Psychiatry Res 2012;200:843-8.

71 Stockings E, Degenhardt L, Lee YY, et al. Symptom screening scales for detecting major depressive disorder in children and adolescents: a systematic review and meta-analysis of reliability, validity and diagnostic utility. J Affect Disord 2015;174:447-63.

72 Löwe B, Kroenke K, Gräfe K. Detecting and monitoring depression with a two-item questionnaire (PHQ-2). J Psychosom Res 2005;58:163-71.

73 Klein EM, Brähler E, Dreier M, et al. The German version of the Perceived Stress Scale - psychometric characteristics in a representative German community sample. BMC Psychiatry 2016;16:159.

74 Conway M, Csank PA, Holm SL, et al. On assessing individual differences in rumination on sadness. J Pers Assess 2000;75:404-25.

75 Stöber J. The social desirability Scale-17 (SDS-17): convergent validity, discriminant validity, and relationship with age. Eur J Psychol Assess 2001;17:222-32. 